\title{
Analytical solution of linear and nonlinear fractional differential equations
}

\author{
E. A. A. Ziada \\ Nile Higher Institute for Engineering and Technology, Mansoura, Egypt. \\ engemanziada@yahoo.com
}

\begin{abstract}
In this paper, we apply the Adomian decomposition method (ADM) for solving linear and nonlinear fractional differential equations (FDEs). The existence and uniqueness of the solution are proved. The convergence of the series solution and the error analysis are discussed. Some applications are solved such as relaxation-oscillation equation, Basset problem and fractional Riccati differential equation.
\end{abstract}

Keywords: Adomian Method; existence; uniqueness; error analysis; relaxation-oscillation equation; Basset problem; Riccati differential equation.

\section{Introduction}

In this paper, we are concerned with studying the following FDE:

subject to the initial conditions,

$$
D^{\alpha} y(t)+g(t) f(y(t))=x(t), \quad \alpha \in(n-1, n)
$$

$$
\left.\boldsymbol{D}^{\alpha-k} y(t)\right|_{t=0}=0, \quad k=1,2, \ldots, n .
$$

where $\boldsymbol{D}^{\alpha}$ is Riemann-Liouville fractional derivative, the proof of convergence and error analysis of ADM when applied to problem (1)-(2), is introduced. The linear case is a special case from the nonlinear case; So we solve some linear and nonlinear numerical examples and comparing the results with another traditional methods. The existence and uniqueness of the solution $y \in$ $C(J)$,where $C(J)$ is the space of all continuous functions and $J=[0, T], T<\infty$ of the problem (1)-(2) will be proved, the integral representation of this solution will be proved, the solution algorithm and the convergence of the series solution using ADM will be discussed. Some 
applications are given such as relaxation-oscillation equation, Basset problem and fractional Riccati differential equation

\section{The solution algorithm}

Applying the integral operator of order $\alpha$ to the problem (1)-(2), this reduces it to its equivalent fractional integral equation (FIE):

$$
\begin{gathered}
y(t)=\frac{1}{\Gamma(\alpha)} \int_{0}^{t}(t-\tau)^{\alpha-1} x(\tau) d \tau-\frac{1}{\Gamma(\alpha)} \int_{0}^{t} g(\tau)(t \\
-\tau)^{\alpha-1} f(y(\tau)) d \tau
\end{gathered}
$$

Assume that $x(t)$ is bounded $\forall t \in I=[0, T], T \in R^{+},|g(\tau)| \leq M \forall 0 \leq \tau \leq t \leq T, M$ is a positive finite constant, $y \in C[I]$, and $f(y)$ satisfies Lipschitz condition with Lipschitz constant $L$ such as,

and has Adomian polynomials representation,

$$
|f(y)-f(z)| \leq L|y-z|
$$

$$
f(y)=\sum_{n=0}^{\infty} A_{n}\left(y_{0}, y_{1}, \ldots, y_{n}\right)
$$

where,

$$
A_{n}=\frac{1}{n !} \frac{d^{n}}{d \lambda^{n}}\left[f\left(\sum_{i=0}^{\infty} \lambda^{i} y_{i}\right)\right]_{\lambda=0}
$$

Substitute from equation (5) into equation (3) we get,

$$
\begin{gathered}
y(t)=\frac{1}{\Gamma(\alpha)} \int_{0}^{t}(t-\tau)^{\alpha-1} x(\tau) d \tau-\frac{1}{\Gamma(\alpha)} \int_{0}^{t} g(\tau)(t \\
-\tau)^{\alpha-1} \sum_{n=0}^{\infty} A_{n} d \tau
\end{gathered}
$$

Let $y(t)=\sum_{n=0}^{\infty} y_{n}(t)$ in (7) and applying ADM, we get the following recursive relations,

$$
\begin{gathered}
y_{0}(t)=\frac{1}{\Gamma(\alpha)} \int_{0}^{t}(t-\tau)^{\alpha-1} x(\tau) d \tau \\
y_{i}(t)=-\frac{1}{\Gamma(\alpha)} \int_{0}^{t} g(\tau)(t-\tau)^{\alpha-1} A_{i-1} d \tau, \quad i \geq 1 .
\end{gathered}
$$

Finally, the solution is,

$$
y(t)=\sum_{i=0}^{\infty} y_{i}(t)
$$

\section{Convergence analysis}

\subsection{Existence and uniqueness of the solution}

Define the mapping $F: E \rightarrow E$ where $E$ is the Banach space $(C[I],\|\cdot\|)$, the space of all 
continuous functions on $I$ with the norm $\|f(t)\|=\max _{t \in I}|f(t)|$.

\section{Theorem 1:}

The problem (1)-(2) has a unique solution whenever $0<\beta<1$ where $\beta=\frac{L M T^{\alpha}}{\Gamma(\alpha+1)}$.

\section{Proof:}

The mapping $F: E \rightarrow E$ is defined as,

$$
F y(t)=\frac{1}{\Gamma(\alpha)} \int_{0}^{t}(t-\tau)^{\alpha-1} x(\tau) d \tau-\frac{1}{\Gamma(\alpha)} \int_{0}^{t} g(\tau)(t-\tau)^{\alpha-1} f(y(\tau)) d \tau
$$

Let $y(t), z(t) \in E$ :

$$
\begin{aligned}
& \|F y-F z\|=\max _{t \in I}\left|\frac{1}{\Gamma(\alpha)} \int_{0}^{t} g(\tau)(t-\tau)^{\alpha-1}[f(y)-f(z)] d \tau\right| \\
& \quad \leq \frac{1}{\Gamma(\alpha)} \max _{t \in I} \int_{0}^{t}(t-\tau)^{\alpha-1}|g(\tau)||f(y)-f(z)| d \tau \\
& \leq \frac{L M}{\Gamma(\alpha)} \max _{t \in I}|y-z| \int_{0}^{t}(t-\tau)^{\alpha-1} d \tau \\
& \leq \frac{L M T^{\alpha}}{\Gamma(\alpha+1)}\|y-z\| \\
& \leq \beta\|y-z\|
\end{aligned}
$$

Under the condition $0<\beta<1$, the mapping $F$ is contraction [1] and hence there exists a unique solution of the problem (1)-(2) and this completes the proof.

\subsection{Proof of convergence}

\section{Theorem 2:}

The series solution (10) of the problem (1)-(2) using ADM converges if $\left|y_{1}\right|<\infty$ and $0<\beta<$ $1, \beta=\frac{L M T^{\alpha}}{\Gamma(\alpha+1)}$.

Proof:

Define the sequence $\left\{S_{n}\right\}$ such that, $S_{n}=\sum_{i=0}^{n} y_{i}(t)$ is the sequence of partial sums from the series solution $\sum_{i=0}^{\infty} y_{i}(t)$ since,

so,

$$
f(y)=f\left(\sum_{i=0}^{\infty} y_{i}(t)\right)=\sum_{i=0}^{\infty} A_{i}\left(y_{0}, y_{1}, \ldots, y_{i}\right)
$$

$$
f\left(S_{n}\right)=\sum_{i=0}^{n} A_{i}\left(y_{0}, y_{1}, \ldots, y_{i}\right)
$$

Let $S_{n}$ and $S_{m}$ be two arbitrary partial sums with $n>m$. Now, we are going to prove that $\left\{S_{n}\right\}$ is a Cauchy sequence in this Banach space.

$$
\left\|S_{n}-S_{m}\right\|=\max _{t \in I}\left|S_{n}-S_{m}\right|=\max _{t \in I}\left|\sum_{i=m+1}^{n} y_{i}(t)\right|
$$


Let $n=m+1$ then,

$$
\begin{aligned}
& =\max _{t \in I}\left|\sum_{i=m+1}^{n}-\frac{1}{\Gamma(\alpha)} \int_{0}^{t} g(\tau)(t-\tau)^{\alpha-1} A_{i-1} d \tau\right| \\
& =\max _{t \in I}\left|\frac{1}{\Gamma(\alpha)} \int_{0}^{t} g(\tau)(t-\tau)^{\alpha-1} \sum_{i=m}^{n-1} A_{i} d \tau\right| \\
& =\max _{t \in I}\left|\frac{1}{\Gamma(\alpha)} \int_{0}^{t} g(\tau)(t-\tau)^{\alpha-1}\left[f\left(S_{n-1}\right)-f\left(S_{m-1}\right)\right] d \tau\right| \\
& \leq \frac{1}{\Gamma(\alpha)} \max _{t \in I} \int_{0}^{t}(t-\tau)^{\alpha-1}\left|g(\tau) \| f\left(S_{n-1}\right)-f\left(S_{m-1}\right)\right| d \tau \\
& \leq \frac{L M}{\Gamma(\alpha)} \max _{t \in I}\left|S_{n-1}-S_{m-1}\right| \int_{0}^{t}(t-\tau)^{\alpha-1} d \tau \\
& \left\|S_{n}-S_{m}\right\| \leq \frac{L M T^{\alpha}}{\Gamma(\alpha+1)}\left\|S_{n-1}-S_{m-1}\right\| \\
& \leq \beta\left\|S_{n-1}-S_{m-1}\right\|
\end{aligned}
$$

$$
\left\|S_{m+1}-S_{m}\right\| \leq \beta\left\|S_{m}-S_{m-1}\right\| \leq \beta^{2}\left\|S_{m-1}-S_{m-2}\right\| \leq \cdots \leq \beta^{m}\left\|S_{1}-S_{0}\right\|
$$

From the triangle inequality we have,

$$
\begin{aligned}
\left\|S_{n}-S_{m}\right\| & \leq\left\|S_{m+1}-S_{m}\right\|+\left\|S_{m+2}-S_{m+1}\right\|+\cdots+\left\|S_{n}-S_{n-1}\right\| \\
& \leq\left[\beta^{m}+\beta^{m+1}+\cdots+\beta^{n-1}\right]\left\|S_{1}-S_{0}\right\| \\
& \leq \beta^{m}\left[1+\beta+\cdots+\beta^{n-m-1}\right]\left\|S_{1}-S_{0}\right\| \\
& \leq \beta^{m}\left[\frac{1-\beta^{n-m}}{1-\beta}\right]\left\|y_{1}(t)\right\|
\end{aligned}
$$

Since, $0<\beta<1$, and $n>m$ then, $\left(1-\beta^{n-m}\right) \leq 1$. Consequently,

$$
\begin{aligned}
& \left\|S_{n}-S_{m}\right\| \leq \frac{\beta^{m}}{1-\beta}\left\|y_{1}(t)\right\| \\
& \leq \frac{\beta^{m}}{1-\beta} \max _{t \in I}\left|y_{1}(t)\right|
\end{aligned}
$$

but, $\left|y_{1}(t)\right|<\infty$ and as $m \rightarrow \infty$ then, $\left\|S_{n}-S_{m}\right\| \rightarrow 0$ and hence, $\left\{S_{n}\right\}$ is a Cauchy sequence in this Banach space so, the series $\sum_{n=0}^{\infty} y_{n}(t)$ converges and this completes the proof.

\subsection{Error analysis}

For ADM, we can estimate the maximum absolute truncated error of Adomian's series solution in the following theorem.

Theorem 3: The maximum absolute truncation error of the series solution (10) to the problem (1)-(2) is estimated to be,

$$
\max _{t \in I}\left|y(t)-\sum_{i=0}^{m} y_{i}(t)\right| \leq \frac{\beta^{m}}{1-\beta} \max _{t \in I}\left|y_{1}(t)\right|
$$

Proof: From Theorem 2 we have, 


$$
\left\|S_{n}-S_{m}\right\| \leq \frac{\beta^{m}}{1-\beta} \max _{t \in I}\left|y_{1}(t)\right|
$$

but, $S_{n}=\sum_{i=0}^{n} y_{i}(t)$ as $n \rightarrow \infty$ then, $S_{n} \rightarrow y(t)$ so,

$$
\left\|y(t)-S_{m}\right\| \leq \frac{\beta^{m}}{1-\beta} \max _{t \in I}\left|y_{1}(t)\right|
$$

so, the maximum absolute truncation error in the interval $I$ is,

and this completes the proof.

$$
\max _{t \in I}\left|y(t)-\sum_{i=0}^{m} y_{i}(t)\right| \leq \frac{\beta^{m}}{1-\beta} \max _{t \in I}\left|y_{1}(t)\right|
$$

\section{Numerical Examples}

\subsection{Examples for linear FDEs:}

\subsubsection{Application (1): Relaxation-oscillation equation}

Consider the following linear FDE,

$$
\begin{gathered}
{ }_{0} \boldsymbol{D}_{t}^{\alpha} y(t)+A y(t)=f(t), \quad t>0, \quad n-1<\alpha \leq n, \\
\left.\boldsymbol{D}^{\alpha-k} y(t)\right|_{t=0}=0, \quad k=1,2, \ldots, n .
\end{gathered}
$$

For $0<\alpha \leq 2$ this equation is called the relaxation-oscillation equation. In [7] and [3], this problem was solved by the numerical methods, while in [4] was solved by ADM as a system.

Applying the integral operator $J^{\alpha}$ where $\left(J^{\alpha}={ }_{0} \boldsymbol{D}_{t}^{-\alpha}\right)$ on both sides of equation (11) and taking $A=1$ and $f(t)=H(t)$. We get,

$$
\begin{aligned}
& J^{\alpha}\left({ }_{0} \boldsymbol{D}_{t}^{\alpha} y(t)\right)=J^{\alpha}[H(t)]-J^{\alpha} y(t), \\
& y(t)=J^{\alpha}[H(t)]-J^{\alpha} y(t),
\end{aligned}
$$

Applying ADM to the equation (12) we have,

$$
\begin{gathered}
y_{0}(t)=J^{\alpha}[H(t)], \\
y_{n}(t)=-J^{\alpha} y_{n-1}(t), \quad n \geq 1 .
\end{gathered}
$$

The results of our computations for different values of $\alpha(1 \leq \alpha \leq 2)$, are shown in figures 1.a-1.f $(n=50)$. We see from these figures that the solution is a damped oscillator whenever $1<\alpha<2$, and it will be perfect oscillator if $\alpha=2$. These results are in perfect agreement with the analytical solutions; obtained with the help of fractional Green's function for a two-term fractional differential equation. This solution is:

$$
\begin{aligned}
y(t) & =\int_{0}^{t} G_{2}(t-\tau) f(\tau) d \tau, \\
G_{2}(t) & =t^{\alpha-1} E_{\alpha, \alpha}\left(-t^{\alpha}\right), f(t)=H(t) .
\end{aligned}
$$

then,

$$
y(t)=t^{\alpha} E_{\alpha, \alpha+1}\left(-t^{\alpha}\right)
$$




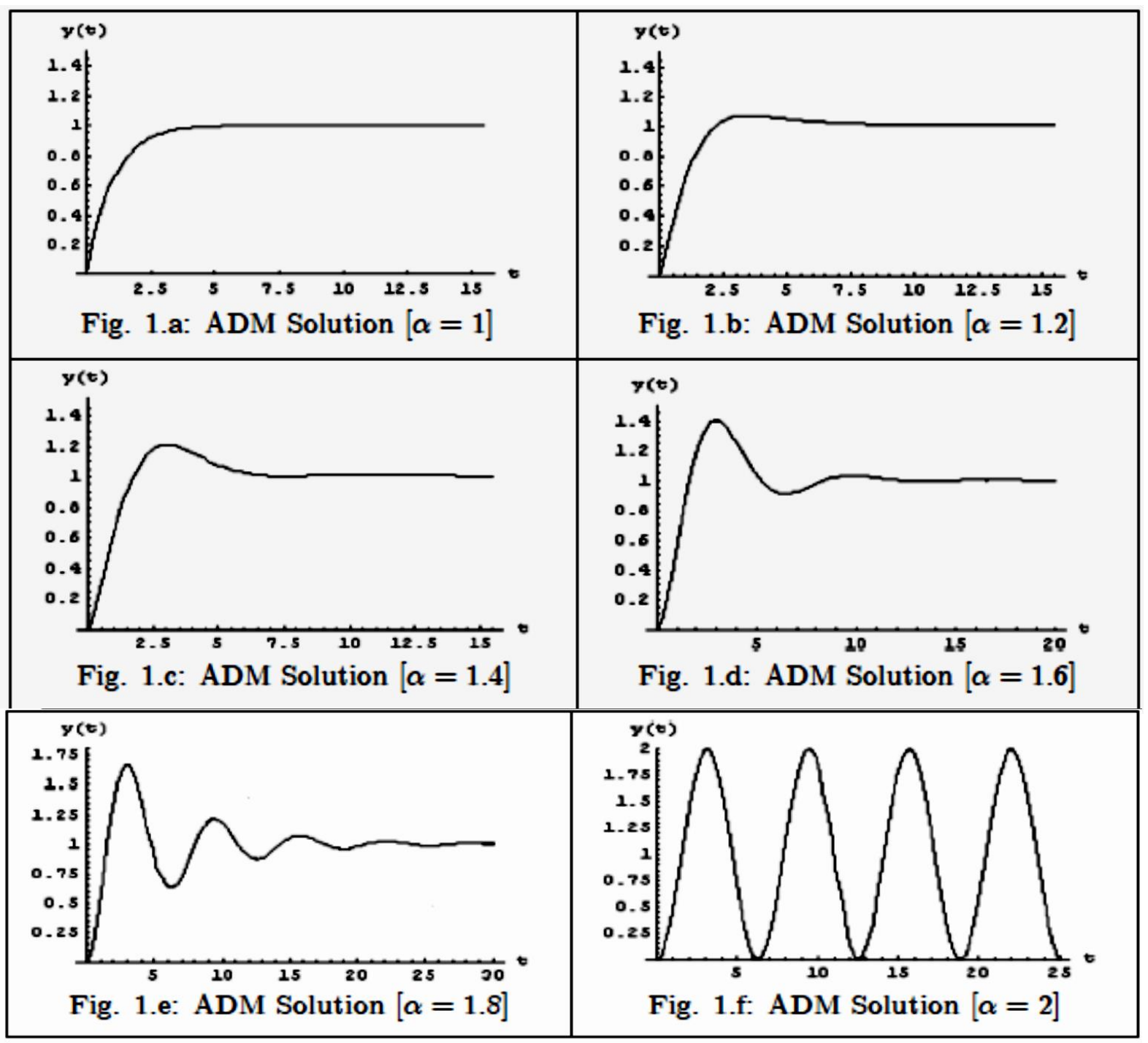

Using the relation (13), the first four-terms of the series solution $(\alpha=3 / 2)$ are,

$$
y(t)=\frac{t^{3 / 2}}{\Gamma(5 / 2)}-\frac{t^{3}}{\Gamma(4)}+\frac{t^{9 / 2}}{\Gamma(11 / 2)}-\frac{t^{6}}{\Gamma(7)}+\cdots
$$

A comparison between ADM and Green's solutions given in Fig. $2(n=50)$. 


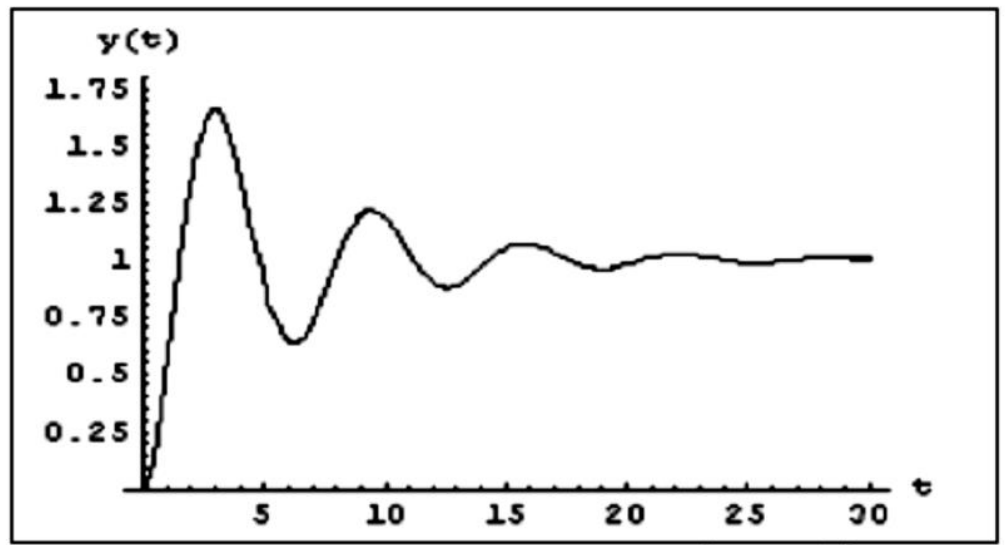

Fig. 2: ADM and Green's Sol. $[\alpha=3 / 2]$

\subsubsection{Basset problem}

Consider the following linear FDE,

$$
\begin{gathered}
\mathbf{D} u(t)+2 a \boldsymbol{D}^{1 / 2} u(t)+b u(t)=q(t), \\
\left.\boldsymbol{D}^{-1 / 2} u(t)\right|_{t=0}=0,
\end{gathered}
$$

where $a$ is a positive constant. If $b=1$, then the linear FDE (16) is called Basset problem, a classical problem in fluid dynamics concerning the unsteady motion of a particle accelerating in a viscous fluid under the action of the gravity [5].

The Laplace transform solution of this problem [6] when ( $a=b=q(t)=1)$ is:

$$
u(t)=1-\left((1-2 t) E_{1 / 2}(-\sqrt{t})+2 \sqrt{t / \pi}\right)
$$

Using ADM to equation (17), we get

$$
\begin{gathered}
u_{0}=t \\
u_{n}=-2 J^{1 / 2}\left[u_{n-1}(t)\right]-J^{1}\left[u_{n-1}(t)\right], \quad n \geq 1 .
\end{gathered}
$$

From the relation (18), the first three-terms of the series solution are:

$$
u(t)=t-\frac{8 t^{3 / 2}}{3 \sqrt{\pi}}-\frac{t^{2}}{2}+\frac{1}{30} t^{2}\left(60+\frac{64 \sqrt{t}}{\sqrt{\pi}}+5 t\right)+\cdots .
$$

Figures 3.a and 3.b show a comparison between ADM solution and Laplace solution. 


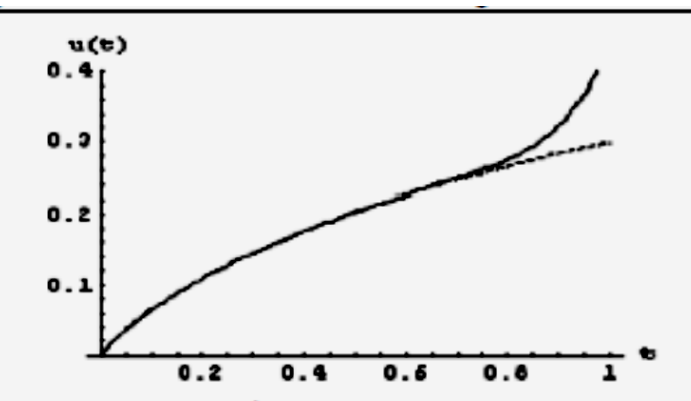

Fig. 3.a: ADM and Laplace Sol.

$$
[n=20]
$$

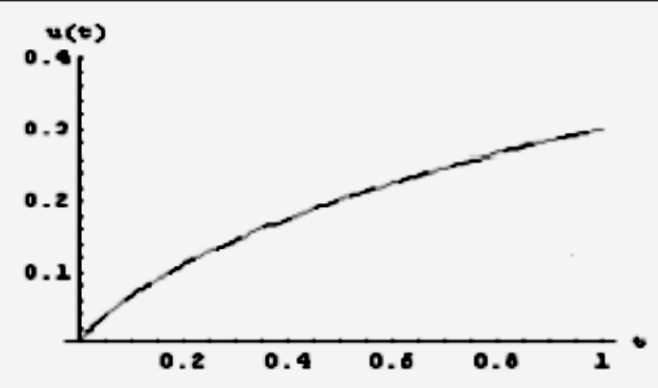

Fig. 3.b: ADM and Laplace Sol.

\subsection{Examples for Nonlinear FDEs}

Example 1. Consider the initial value problem,

$$
\begin{gathered}
\boldsymbol{D}^{\mu} y=y^{2}+1, \quad m-1<\mu \leq m, 0<t<1, \\
\left.\boldsymbol{D}^{\mu-k} y(t)\right|_{t=0}=0, \quad k=1,2, \ldots, m .
\end{gathered}
$$

Operating with $J^{\mu}$ on both sides of equation (20) and using the initial condition (21) we obtain,

$$
y(t)=J^{\mu}[1]+J^{\mu}\left[y^{2}\right]
$$

Use ADM and replace the nonlinear term $f(y)=y^{2}$ by its corresponding Adomian polynomials we have,

$$
\begin{gathered}
y_{0}=J^{\mu}[1], \quad n \geq 1 . \\
y_{n}=J^{\mu}\left[A_{n-1}\right], \quad n \geq 1
\end{gathered}
$$

from the two relations (23) and (24), the six-terms approximation are,

where, the coefficients are given by,

$$
\phi_{6}=\sum_{k=0}^{5} C_{k} t^{(2 k+1) \mu}=1,
$$

$$
\begin{aligned}
& C_{0}=\frac{1}{\Gamma(\mu+1)}, \quad C_{1}=\frac{\Gamma(2 \mu+1)}{\Gamma(3 \mu+1)} C_{0}^{2}, \\
& C_{2}=\frac{\Gamma(4 \mu+1)}{\Gamma(5 \mu+1)}\left(2 C_{0} C_{1}\right), \quad C_{3}=\frac{\Gamma(6 \mu+1)}{\Gamma(7 \mu+1)}\left(2 C_{0} C_{2}+C_{1}^{2}\right), \\
& C_{4}=\frac{\Gamma(8 \mu+1)}{\Gamma(9 \mu+1)}\left(2 C_{0} C_{3}+2 C_{1} C_{2}\right), \quad C_{5}=\frac{\Gamma(10 \mu+1)}{\Gamma(11 \mu+1)}\left(2 C_{0} C_{4}+2 C_{1} C_{3}+C_{2}^{2}\right) .
\end{aligned}
$$

The solution of the problem (20)-(21) by using the numerical method given in [7] is:

$$
h^{-\mu} \sum_{j=0}^{n} w_{j}^{(\mu)} y_{n-j}-y_{n}^{2}=1,
$$

where, $t_{n}=n h, y_{n}=y\left(t_{n}\right), w_{j}^{(\mu)}=(-1)^{j}\left(\begin{array}{c}\mu \\ j\end{array}\right),(n, j=0,1,2, \ldots)$. Therefore, we get

then,

$$
h^{-\mu} \sum_{j=0}^{n}(-1)^{j} \frac{\Gamma(\mu+1)}{\Gamma(j+1) \Gamma(\mu-j+1)} y_{n-j}-y_{n}^{2}=1,
$$




$$
y_{n}=h^{\mu}+h^{\mu} y_{n-1}^{2}-\sum_{j=1}^{n}(-1)^{j} \frac{\Gamma(\mu+1)}{\Gamma(j+1) \Gamma(\mu-j+1)} y_{n-j}
$$

Figures 4.a-4.f illustrate a comparison between ADM solution $(n=5)$ and the numerical solution $(h=0.01)$. For $\mu=0.5$; the numerical method gives unbounded solution when $t \in$ $[0,1]$, see Fig. 4.a, while, ADM gives a bounded solution in the same interval, see Fig. 4.b.

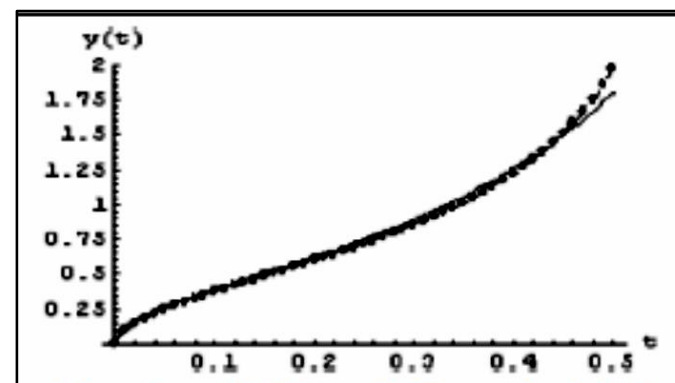

Fig. 4.a: ADM and Numerical Sol.

$$
[\mu=0.5]
$$

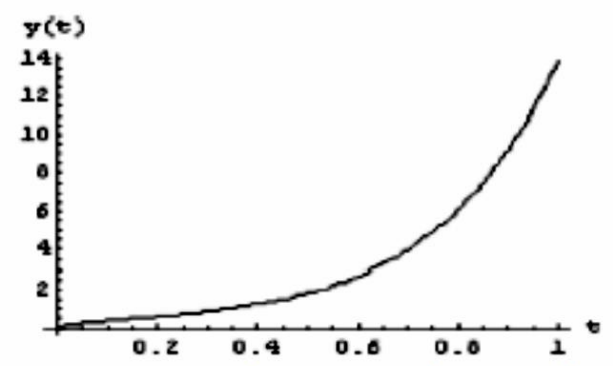

Fig. 4.b: ADM Sol. $[\mu=0.5]$

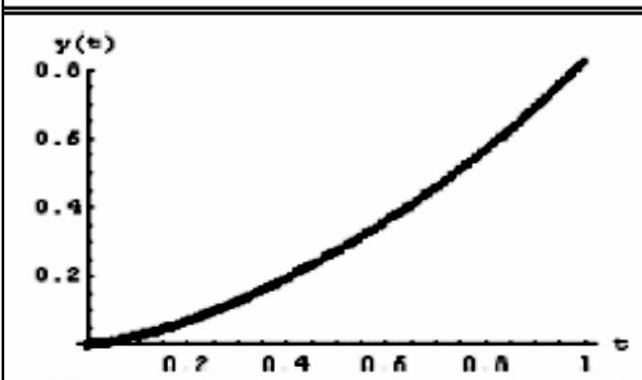

Fig. 4.c: ADM and Numerical Sol.

$$
[\mu=1.5]
$$

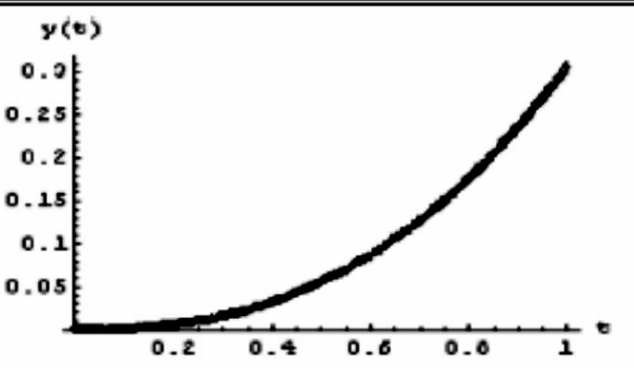

Fig. 4.d: ADM and Numerical Sol.

$$
[\mu=2.5]
$$

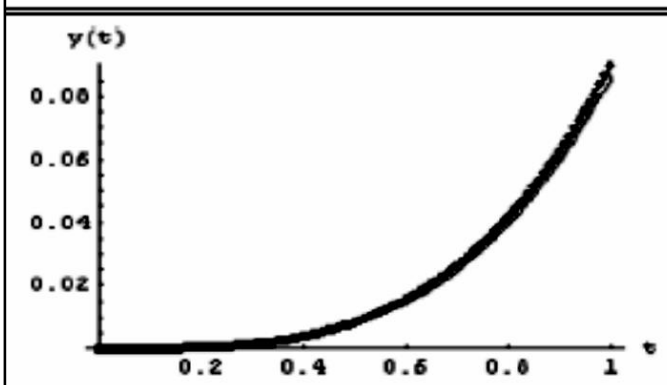

Fig. 4.e: ADM and Numerical Sol.

$$
[\mu=3.5]
$$

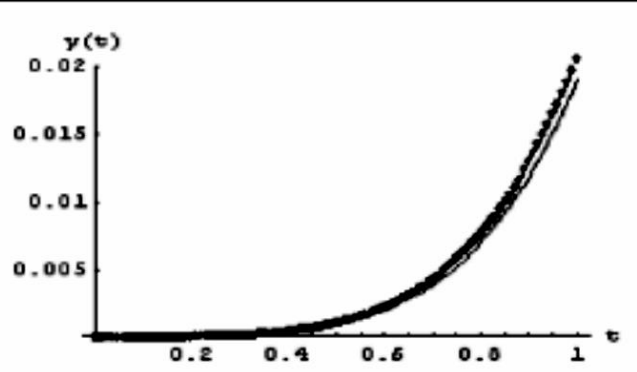

Fig. 4.f: ADM and Numerical Sol.

$$
[\mu=4.5]
$$

Table (3.1) shows the relative error between exact and ADM solution of $\mu=1$. The value $\mu=1$ (ODE) is the only case for which we know the exact solution $(y=\tan t)$ and our approximate solution is in good agreement with the exact values. 
Table (3.1): Relative error $(\mu=1)$

\begin{tabular}{|c|c|c|c|}
\hline$t$ & Exact solution & ADM solution & Relative error \\
\hline 0.1 & 0.100334672 & 0.100334672 & $3.3195594351 \times 10^{-15}$ \\
\hline 0.2 & 0.2027100355 & 0.2027100355 & $1.4756006633 \times 10^{-11}$ \\
\hline 0.3 & 0.3093362496 & 0.309336249 & $1.921474304 \times 10^{-9}$ \\
\hline 0.4 & 0.42279321874 & 0.42279319296 & $6.097058134 \times 10^{-8}$ \\
\hline 0.5 & 0.54630248984 & 0.54630200191 & $8.931495255 \times 10^{-7}$ \\
\hline 0.6 & 0.68413680834 & 0.68413131533 & $8.0291143934 \times 10^{-6}$ \\
\hline 0.7 & 0.84228838046 & 0.84224495232 & 0.0000515597 \\
\hline 0.8 & 1.02963855705 & 1.02937191571 & 0.000258966 \\
\hline 0.9 & 1.26015821755 & 1.25879891374 & 0.001078677 \\
\hline 1.0 & 1.55740772466 & 1.5513676447 & 0.003878291 \\
\hline
\end{tabular}

Example 2. Consider the nonlinear FDE,

$$
\begin{gathered}
\boldsymbol{D}^{5 / 2} y=\frac{1}{4} y^{4}+t, \quad 0<t \leq 1, \\
\left.\boldsymbol{D}^{3 / 2} y\right|_{t=0}=\left.\boldsymbol{D}^{1 / 2} y\right|_{t=0}=\left.\boldsymbol{D}^{-1 / 2} y\right|_{t=0}=0 .
\end{gathered}
$$

Using $\mathrm{ADM}$ to the equation (27), we get

$$
\begin{gathered}
y_{0}=J^{5 / 2}(t), \\
y_{n}=\frac{1}{4} J^{5 / 2}\left(A_{n-1}\right), \quad n \geq 1 .
\end{gathered}
$$

From the relations (28) and (29), the first two-terms of the series solution are,

$$
y(t)=\left(\frac{16 t^{7 / 2}}{105 \sqrt{\pi}}\right)+\left(\frac{274877906944 t^{33 / 2}}{2009196669692953125 \pi^{3 / 2}}\right)+\cdots .
$$

Figures 5.a-5.d show ADM solution of problem (27) at different values of $m$.

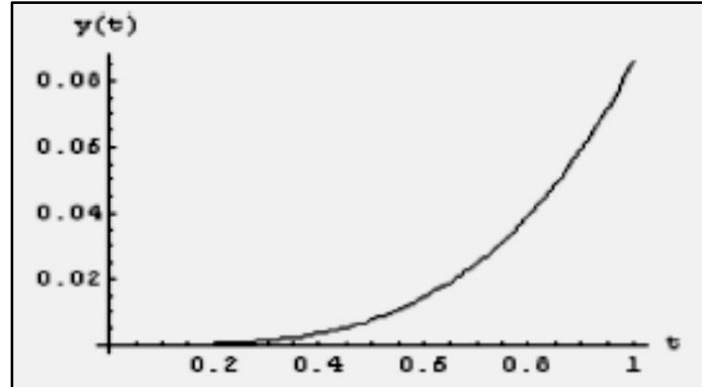

Fig. 5.a: ADM Sol. $[m=5]$

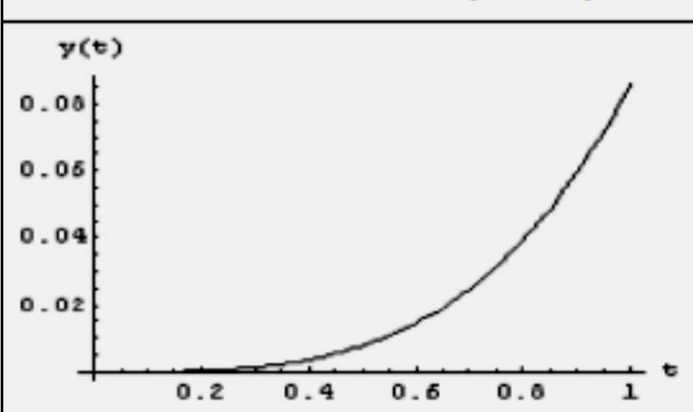

Fig. 5.c: ADM Sol. $[m=15]$

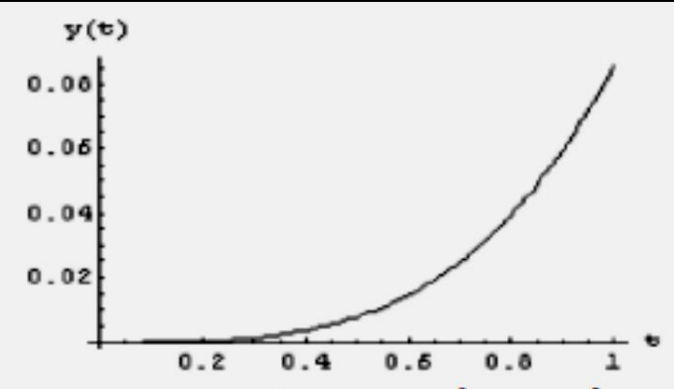

Fig. 5.b: ADM Sol. $[m=10]$

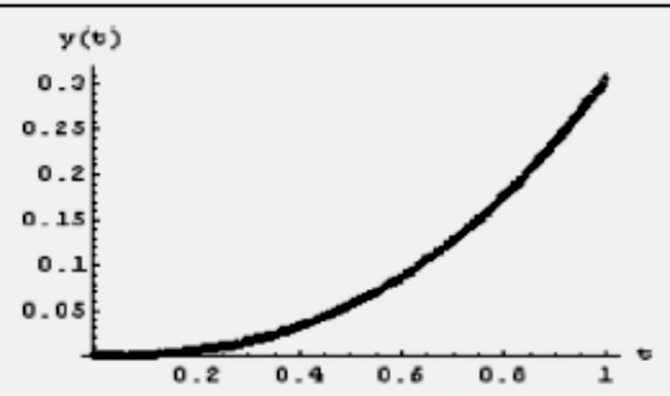

Fig. 5.d: ADM Sol. $[m=20]$ 
Now, we will evaluate the maximum absolute truncated error of the series solution (30).

- $\quad$ Lipschitz constant $(L)$ :

$$
\begin{aligned}
|f(y)-f(z)| & =\left|y^{4}-z^{4}\right| \\
& =\left|y^{2}+z^{2}\right||y+z||y-z| \\
& \leq 4|y-z| \Rightarrow L=4 .
\end{aligned}
$$

The maximum error:

- $M \geqq|g(\tau)| \leq \frac{1}{4} \Rightarrow M=\frac{1}{4}$.

- $\beta$ $\quad \beta=\frac{L M T^{\alpha}}{\Gamma(\alpha+1)}=\frac{1}{\Gamma(7 / 2)}$.

- $\max _{t \in I}\left|y_{1}(t)\right|=\frac{274877906944}{2009196669692953125 \pi^{3 / 2}}$.

i. For $m=5: \quad \max _{t \in I}\left|y(t)-\sum_{i=0}^{5} y_{i}(t)\right| \leq 8.66908 \times 10^{-11}$.

ii. For $m=10: \max _{t \in I}\left|y(t)-\sum_{i=0}^{10} y_{i}(t)\right| \leq 2.13841 \times 10^{-13}$.

iii. For $m=15: \max _{t \in I}\left|y(t)-\sum_{i=0}^{15} y_{i}(t)\right| \leq 5.27486 \times 10^{-16}$.

iv. For $m=20: \max _{t \in I}\left|y(t)-\sum_{i=0}^{20} y_{i}(t)\right| \leq 1.30116 \times 10^{-18}$.

\subsubsection{Application: Fractional Riccati differential equation}

The fractional Riccati differential equation,

$$
\begin{gathered}
\boldsymbol{D}^{\alpha} y+y^{2}=1, \quad k-1<\alpha \leq k, 0<t \leq 0.5 \\
\left.\boldsymbol{D}^{\alpha-i} y(t)\right|_{t=0}=0, \quad i=1,2, \ldots, k
\end{gathered}
$$

was solved before using the numerical methods in [8]-[10].

Applying ADM to equation (31) and using the given initial conditions, we get

$$
\begin{gathered}
y_{0}=J^{\alpha}(1), \\
y_{n}=-J^{\alpha}\left(A_{n-1}\right), \quad n \geq 1 .
\end{gathered}
$$

From the relation (32), the first three-terms of the series solution at $(\alpha=1 / 2)$ are,

$$
y(t)=\left(\frac{2 \sqrt{t}}{\sqrt{\pi}}\right)+\left(\frac{-16 t^{3 / 2}}{3 \pi^{3 / 2}}\right)+\left(\frac{1024 t^{5 / 2}}{45 \pi^{5 / 2}}\right)+\cdots
$$

while, the solution algorithm in this case using the numerical method given in [7] is:

$$
y_{n}=h^{1 / 2}-h^{1 / 2} y_{n-1}^{2}-\sum_{j=1}^{n}(-1)^{j} \frac{\Gamma(3 / 2)}{\Gamma(j+1) \Gamma(3 / 2-j)} y_{n-j}
$$

where, $t_{n}=n h, y_{n}=y\left(t_{n}\right),(n, j=0,1,2, \ldots)$.

The exact solution of this problem at $(\alpha=1)$ is $y(t)=\frac{e^{2 t}-1}{e^{2 t}+1}$, while ADM series solution is:

$$
y(t)=t-\frac{t^{3}}{3}+\frac{2 t^{5}}{15}+\cdots
$$

A comparison between ADM solution $(\alpha=1 / 2, n=9)$ and numerical solution $(h=0.01)$ is given in Fig. 6.a, while Fig. 6.b shows a comparison between ADM solution $(\alpha=1, n=5)$ and exact solution. 


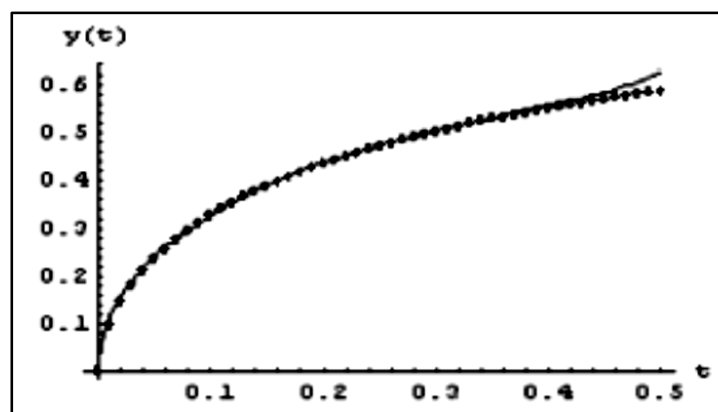

Fig. 6.a: ADM and Numerical Sol.

$$
[\alpha=1 / 2]
$$

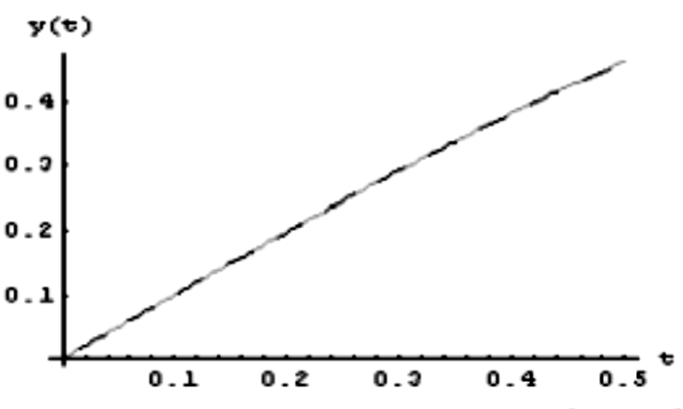

Fig. 6.b: ADM and Exact Sol. $[\alpha=1]$

From the relation (32), the first three-terms of ADM series solution at $(\alpha=3 / 2)$ is,

$$
y(t)=\frac{4 t^{3 / 2}}{3 \sqrt{\pi}}-\frac{1024 t^{9 / 2}}{2835 \pi^{3 / 2}}+\frac{33554432 t^{15 / 2}}{383107725 \pi^{5 / 2}}-\cdots .
$$

A comparison between $\mathrm{ADM}$ solution $(\alpha=3 / 2, n=5)$ and numerical solution $(h=0.01)$ is given in Fig. 6.c.

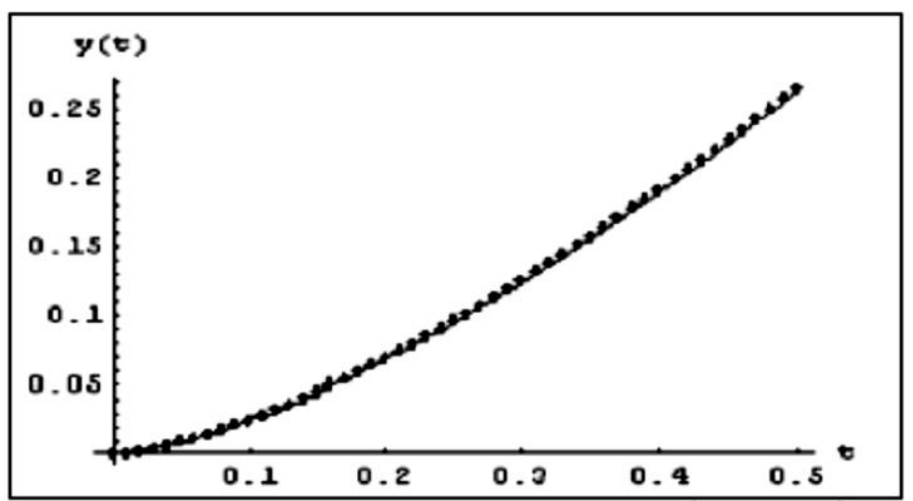

Fig. 6.c: ADM and Numerical Sol. $[\alpha=3 / 2]$

Now, we will evaluate the maximum absolute truncated error of the series solution (36).

The maximum error:

i. For $m=5: \quad \max _{t \in I}\left|y(t)-\sum_{i=0}^{5} y_{i}(t)\right| \leq 5.19711 \times 10^{-6}$.

ii. For $m=10: \max _{t \in I}\left|y(t)-\sum_{i=0}^{10} y_{i}(t)\right| \leq 6.91602 \times 10^{-9}$.

iii. For $m=15: \quad \max _{t \in I}\left|y(t)-\sum_{i=0}^{15} y_{i}(t)\right| \leq 9.20344 \times 10^{-12}$.

iv. For $m=20: \max _{t \in I}\left|y(t)-\sum_{i=0}^{20} y_{i}(t)\right| \leq 1.22474 \times 10^{-14}$.

\section{References}

[1] E. Kreyszig, Introductory Functional Analysis with Applications, New York, (1975).

[2] I. Podlubny, Fractional differential equations, Academic Press, New York, 1999.

[3] G. Adomian, Nonlinear Stochastic Operator Equations, Academic press, San Diego,1986. 
[4] I. Podlubny, Solution of linear fractional differential equations with constant coefficients, in: P. Rusey, I. Dimovski, V. Kiryakova (eds.), Transform Methods and Special Functions, SCT Publishers, Singapore, 1995, pp. 217-228.

[5] F. Mainardi, Fractional calculus: some basic problems in continuum and statistical mechanics, in: A. Carpinteri, F. Mainardi (Eds.), Fractals \& Fractional Calculus in Continuum Mechanics, Springer, New York, 1997, pp. 291-348.

[6] K. Diethelm, and N. J. Ford, Numerical Solution of the Bagley-Torvik Equation, BIT 42, (2002), pp. 490-507.

[7] I. Podlubny, Fractional differential equations, Academic Press, New York, 1999.

[8] K. Diethelm, and N. J. Ford, The numerical solution of linear and nonlinear fractional differential equations involving fractional derivatives of several orders, Numerical Analysis Report 379, Manchester Center for Computational Mathematics, Manchester, England, 2001.

[9] C. Trinks, and P. Ruge, Treatment of dynamic systems with fractional derivatives without evaluating memory-integrals, Comput. Mech. 29 (6), (2002), pp. 471-476.

[10] A. Carpinteri, and F. Mainardi, Fractals and Fractional Calculus in Continuum Mechanics, Springer Verlag, Wien and New York 1997, pp. 223-276. 